\title{
Improving Public Health Surveillance methods via Smart Home technologies
}

\author{
Kirti S. Sahu¹, Arlene Oetomo ${ }^{1}$, Plinio P. Morita1, 2, 3 \\ ${ }^{1}$ University of Waterloo, Waterloo, Ontario, Canada, ${ }^{2}$ University of Toronto, Toronto, Ontario, Canada, ${ }^{3}$ Techna institute, Toronto, Ontario, Canada \\ Objective
}

The objective of this study is to explore individual, household and population-level health indicators collected in the home via smart thermostats. The study's approach is to (a) identify if it is possible to isolate specific user behaviours using the motion and thermostat sensor data, and (b) develop Remote Monitoring of healthy behaviours at population level. Furthermore, this study is interested in identifying if observed patterns will suffer variations. As a result, it will be possible to understand human behaviours and consequently understand lifestyle habits of a person or a group of people.

\section{Introduction}

Public health surveillance relies on surveys and/or self-reported data collection, both of which require manpower, time commitment, and financial resources from public health agencies and participants. The survey results can quickly become outdated due to fastpaced changes in our society. The health habits of Canadians have rapidly evolved with technology and research indicates we are becoming a sedentary society, thus the levels of physical activity (PA) are very important population level health indicators. We will present a novel method to gather data at a granular level in near real-time, with minimal effort from participants. Simple thermostats are found in nearly every house in Canada, and smart thermostats enable efficient temperature adjustment, saving energy costs by adjusting according to human activity. Thermostats are ubiquitous in Canadian homes and the current expansion of smart thermostats make them an ideal data source over traditional methods. Utilizing technology that can be deployed at a population level will enable vast granular data collection beyond capabilities of traditional surveys. In this project UbiLab [1] is exploring the use of the zero-effort technology using sensor data collected by smart thermostats and other associated sensors to develop an innovative health surveillance platform and monitor an individual's health at the household level as well as health indicators at population level. Utilizing the smart wi-fi thermostat, we able to report on PA, sedentary behaviour, and sleep patterns at the household level. The thermostat and remote sensors (RS) contain temperature and motion sensors, which can be used to monitor activity in the home (i.e. lack of travel indicates sedentary behaviour), as well as sleep characteristics. This is beneficial as no action is required from participants, allowing individuals to go about their lives unperturbed. This powerful system will be able to deliver real-time health insights to public health professionals.

\section{Methods}

Zero-effort-technologies [2] represent the future of ambient assisted living (AAL), in which sensors gather data generated by the person without conscious effort by the user. Such data could be integrated with other technologies to give the system the ability to tackle unsolved remote monitoring issues challenged the traditional data collection method barriers. For example, when the RS is placed in the bedroom, they can provide insights on sleep duration and quality. This addresses the challenges of declining participant engagement, low response rates in surveys and focus groups, and technical barriers to wearable technology. This eliminates recall bias, common when asking participants to quantify the amount of PA and types of behaviours they engaged in. Using the motion data, we can quantify the amount of PA in the home to determine individual levels of PA. The UbiLab partnered with ecobee [3], a Canadian smart wi-fi thermostat company, leveraging data from over 10,000 households in North-America collected through the Donate Your Data (DYD) [4] program. A small pilot study $(n=8)$ was done to validate the use of motion sensor readings of movement between rooms through a cross comparison with Fitbit [5] step data. And the DYD dataset was analyzed for patterns using Python [6], pandas [7], Elasticsearch [8], and Kibana [8]. This method will enable the delivery of personalized insights to monitor individual- and population- level health behaviours.

\section{Results}

Physical Activity, Sedentary Behaviour and Sleep (PASS) indicators [9] are measured through surveys (i.e. Canadian Health Measures Survey and Canadian Community Housing Survey) administered by Statistics Canada. Using this technology public

SDS Annual Conference Proceedings 2019. This is an Open Access article distributed under the terms of the Creative Commons AttributionNoncommercial 4.0 Unported License (http://creativecommons.org/licenses/by-nc/3.0/), permitting all non-commercial use, distribution, and reproduction in any medium, provided the original work is properly cited. 
health agencies will enable to collect novel health indicators, monitor health in real-time and deliver health insights to Canadians to increase health literacy. A positive association between Fitbit and ecobee data was found (Spearman's Correlation coefficient $=$ 0.7, $\mathrm{p}>0.001$ ) from 380 person hours from the pilot study. Indicators (sleep, interrupted sleep, daily indoor activity, sedentary) based on the PASS Indicators Framework from the Public Health Agency of Canada (PHAC) [2] were measured using DYD data. Single occupant ecobee households in Canada averaged 7.2 hours of sleep in 24-hours, 2.1 hours of interrupted sleep, were active for 85 minutes daily, and spent 4.44 hours being sedentary. Recently, we have improved data collection adding Fitbit Charge 2 HRs, to capture sleep and heart rate not previously possible with the Fitbit Zip. Adding more sensors functionality is crucial for algorithm modifications, this includes collecting additional data via the Samsung SmartThings Hub [10]; presence, light usage, and luminance. ecobee is sharing participants and data from their own study, increasing variability within data. We have improved our data storage and analysis process, moving the big data architecture from python to Elasticsearch for real-time data streaming and analysis. We are also actively collaborating with PHAC and improving our algorithm and analysis process using their feedback.

\section{Conclusions}

This is a key opportunity to innovate traditional data collection methods, empowering patients through education and leveraging technology infrastructures to enable healthcare and policy decisions to be made with relevant and real-time data. Lessons learned at the individual and community health levels will be shared with community members and researchers. Implications include understanding short-term impacts with minimal effort and new health policies at the community level. Increased awareness and improvement can help to better physical activity, sleep and sedentary behaviour which may lead to improvements in overall health and wellbeing.

\section{Acknowledgement}

The UbiLab team would like to thank ecobee and their staff for their assistance throughout this project. Thank you to Dr. Plinio Morita for his guidance and leadership.

\section{References}

1. Waterloo U of. Ubilab. https://uwaterloo.ca/ubiquitous-health-technology-lab/.

2. Public Health Agency of Canada - Canada. ca. https://www.canada.ca/en/public-health.html. Accessed October $26,2018$.

3. ecobee | Smart Home Technology|. https://www.ecobee.com/. Accessed October 26, 2018.

4. Donate your Data / Smart WiFi Thermostats by ecobee. https://www.ecobee.com/donateyourdata/. Accessed September 21, 2017.

5. Fitbit Official Site for Activity Trackers \& More. https://www.fitbit.com/en-ca/home. Accessed September 21, 2017.

6. Welcome to Python.org. https://www.python.org/. Accessed November 22, 2017.

7. Python Data Analysis Library — pandas: Python Data Analysis Library. https://pandas.pydata.org/. Accessed January 14, 2018.

8. Elasticsearch. https://www.elastic.co/. Accessed October 26, 2018.

9. Physical Activity, Sedentary Behaviour and Sleep (PASS) Indicator Framework for surveillance - Canada.ca. https://www.canada.ca/en/services/health/monitoring-surveillance/physical-activity-sedentary-behavioursleep.html. Accessed January 14, 2018.

10. Samsung. Samsung Smart thing hub. 2018. https://www.smartthings.com/products/smartthings-hub. 